Avendaño Rodríguez, K. C., Magaña Medina, D. E. y Flores Crespo, P. (2020). Influencia familiar en la elección de carreras STEM (Ciencia, tecnología, ingeniería y matemáticas) en estudiantes de bachillerato. Revista de Investigación Educativa, 38(2), 515-531.

DOI: http://dx.doi.org/10.6018/rie.366311

\title{
Influencia familiar en la elección de carreras STEM (Ciencia, tecnología, ingeniería y matemáticas) en estudiantes de bachillerato
}

\section{Family Influence on the "Choice of STEM careers" (science, technology, engineering and mathematics) in high school students}

\author{
Karla Cristina Avendaño Rodríguez*, Deneb Elí Magaña Medina** y Pedro Flores Crespo*** \\ *Sin afiliación institucional \\ **División de Ciencias Económico Administrativas DACEA. Universidad Juárez Autónoma de Tabasco. (México) \\ ***Facultad de Ciencias Sociales y Políticas. Universidad Autónoma de Querétaro. (México)
}

\begin{abstract}
Resumen
La sociedad experimenta cambios científicos y tecnológicos que están obligando a las economías a centrar su atención en la formación de capital humano con un perfil en ciencia, tecnología, ingeniería y matemáticas (STEM por sus siglas en inglés). Elegir una carrera es una decisión de tipo individual y familiar, este artículo tiene como objetivo conocer la influencia de la familia (madre o tutora, padre o tutor y hermanos) en la elección de carreras STEM en estudiantes de segundo y sexto semestre de bachillerato. Se entrevistó a 1 mil 759 estudiantes de seis bachilleratos distintos, a través de un muestreo no probabilístico, por cuotas. Los resultados muestran que la madre o tutora es la figura que más influye en la elección de una carrera STEM en comparación con el padre o tutor. Se pensó que los hermanos podrían tener un efecto mayor en los estudiantes, no obstante, los resultados muestran que no es significativo que tengan o no hermanos y que estos puedan influir en la elección de carrera. Sin embargo, es necesario profundizar más en el estudio de los hermanos mayores que egresan de carreras STEM y el efecto que esto tiene en la elección de los hermanos menores, aspecto que no se abordó en la investigación. El estudio no profundizo en variables como: el tipo de carrera y el rol laboral que desempeñan los padres y si está o no relacionado con las áreas STEM. Se concluye que las
\end{abstract}

Correspondencia: Karla Cristina Avendaño Rodríguez, abril_90_04@hotmail.com. 
familias deben realizar actividades no formales que ayuden a despertar el interés en la ciencia y aumentar su capital cultural desde edades tempranas.

Palabras clave: influencia familiar; elección de carreras; estudiantes de bachillerato; educación STEM.

\begin{abstract}
Society experiences scientific and technological changes that are forcing economies to focus their attention on the development of human capital with a profile in science, technology, engineering and mathematics (STEM). This article aims to demonstrate the influence of the family (mother or guardian father or guardian and siblings) on the choice of STEM careers in high school students during their second and sixth semester, since choosing a career is an individual and family decision. A total of 1,759 students from six different high schools were interviewed, through a non-probabilistic sampling, by quotas. The results show that the mother or guardian is the figure that most influences the choice of a STEM career compared to the father or guardian. It was thought that siblings could have a greater effect on students, however, the results show that it is not significant that they have siblings or that siblings can influence their choice. However, it is necessary to go deeper into the study of older brothers who graduate from STEM degrees and the effect this has on the younger brother's election, an aspect that was not addressed in the investigation. The study did not delve into variables such as the type of career and the labor role played by parents and whether or not it is related to STEM areas. In conclusion, families must carry out non-formal activities that help awaken interest in science and increase their cultural capital from an early age.

Keywords: family influence; career choice; high school students; education STEM.
\end{abstract}

\title{
Introducción
}

El término “educación STEM ${ }^{1}$ por sus siglas en inglés (Science, Technology, Engineering \& Mathematics), corresponden en el idioma español a los términos de Ciencia, Tecnología, Ingeniería y Matemáticas (National Academy of Sciences, 2014). En los últimos años ha crecido la demanda de profesionales en estas disciplinas. En 2010, había 7.6 millones de trabajadores STEM en Estados Unidos, representando aproximadamente 1 de cada 18 empleos. Se proyectó que las ocupaciones STEM crecerían en un 17.0 por ciento de 2008 a 2018, en comparación con un crecimiento de 9.8 por ciento para las ocupaciones no STEM (Langdon, McKittrick, Beede, Khan, \& Doms, 2011).

La National Science Foundation (NSF) en Estados Unidos, incluye las categorías comunes de matemáticas, ciencias naturales, ingeniería, ciencias de la computación e informática, también incluye algunas disciplinas asociadas a las ciencias sociales y de la conducta, tales como: psicología, economía, sociología y política (National Science Board, 2016). Es indispensable aumentar el aprendizaje de los estudiantes de las mate-

1 El término se refiere a la educación interrelacionada entre ciencia, tecnología, ingeniería y matemáticas desde preescolar hasta el grado 12, cuya equivalencia en México se refiere a la educación primaria, secundaria y preparatoria. El movimiento busca desarrollar en los estudiantes de todos los niveles educativos habilidades de investigación, pensamiento crítico, solución de problemas, creatividad, comunicación y colaboración. 
rias y prácticas de estas disciplinas y desarrollar actitudes positivas hacia ellas, se ha convertido en uno de los principales objetivos para la educación STEM K-12 en los Estados Unidos (Nadelson \& Seifert, 2017).

Diversos estudios muestran que la sociedad experimenta cambios científicos y tecnológicos que están obligando a las economías a poner atención en la educación STEM desde edades tempranas (Toma \& Greca, 2018; Breiner, Harkness, Johnson \& Koehler, 2012). Así lo muestra el informe "Perspectivas económicas de América Latina educación, competencia e innovación para el desarrollo" publicado en 2015, mostró que el bajo porcentaje de egresados en carreras STEM está generando escasa innovación, limitada formación de capital humano, bajo número de patentes, prototipos, publicación de artículos, generación y transferencia de conocimiento (Organización para la Cooperación y el Desarrollo Económico (OCDE), Banco de Desarrollo de América Latina (CAF) y la Comisión Económica para América Latina y el Caribe (CEPAL), 2015). Mientras que el acelerado crecimiento científico y tecnológico en los países asiáticos se debe principalmente al financiamiento gubernamental y privado, a la implementación de reformas en materia de ciencia y tecnología, a una amplia disponibilidad de mano de obra, además de un sistema educativo que favorece la formación científico-tecnológica desde edades tempranas, y a un alto nivel de alfabetización científica en la sociedad, América Latina está lejos de estos modelos (Han \& Appelbaum, 2018).

México se enfrenta a la Cuarta Revolución Industrial, al igual que el resto del mundo (Rojko, 2017). Y de forma especial sabemos que los países de Latinoamérica aún no han integrado de forma efectiva la Educación en STEM por lo tanto sigue existiendo una fuerte inclinación de los jóvenes por las ciencias sociales (Montgomery \& Fernández-Cárdenas, 2018). Es cierto que la sociedad requiere de perfiles con una profunda formación social, sin embargo es necesario tener claro que la base de las economías esta en la I+D+i (Investigación, Desarrollo e Innovación (Wong, Ho \& Autio, 2005).

Por esta razón, es urgente establecer estrategias de fomento de vocaciones científicas desde la infancia y donde la familia juega un papel particularmente importante (Peterson, Gaskill, \& Cordova, 2018). De acuerdo con Palos \& Drobot, (2010), las variables como la estructura familiar, los roles asumidos por cada miembro, las relaciones entre ellos, el sistema de valores y actitudes que se basan en los comportamientos, influyen en la elección de carrera.

Se preguntó a los estudiantes sobre su composición familiar, el nivel educativo de los padres o tutores y aspectos de tipo socioeconómico. Los resultados son sumamente interesantes en términos de elección de carrera e influencia familiar. El estudió tiene algunas limitaciones tales como: que no se profundizo en la genealogía profesional, en los roles laborales de los padres o tutores, intereses familiares y las relaciones que tienen con las áreas STEM. Esto deberá ser materia de otro estudio empírico.

El artículo está dividido en cuatro partes. La primera, es una revisión de literatura donde se muestra la importancia de la familia en la formación STEM y la importancia del rol materno en dichos procesos. En la segunda parte, se describe la metodología 
empleada, en el tercer apartado se muestran los resultados y en la última parte se presenta la discusión y conclusiones.

\section{La importancia de la familia en la formación STEM}

De acuerdo con Bourdieu (1997), la familia es concebida como un capital cultural, en donde el individuo adquiere un conjunto de cualificaciones producidas por el medio familiar. El autor afirma que la desigualdad del éxito académico no es resultado de la desigualdad económica sino por el capital cultural, el cual implica que el niño lleva desde el seno familiar y antes de ingresar a una escuela, todas las cualidades, capacidades y habilidades que requerirá aplicar en su contexto escolar. Sin embargo, es necesario enfatizar que una buena posición económica proporciona a los hijos mayores y mejores experiencias de vida las cuales contribuyen a incrementar su capital cultural. Por ejemplo, una familia con un nivel socioeconómico bajo y que vive en una zona rural tiene menos probabilidades de realizar experiencias de vida como: visitar un museo científico o tecnológico, hacer excursiones, asistir a talleres infantiles de ciencia, incluso contar con herramientas tecnológicas como: internet, Smart Tv, computadora, tableta electrónica o smartphone, los cuales limitan o incrementan dicho capital cultural. Los resultados de la investigación de Holmes, Gore \& Lloyd (2017), mostraron que poseer un alto capital cultural, ser hombre, tener un padre en una ocupación STEM y un alto rendimiento previo en lectura y aritmética son factores significativos para optar por carreras con componente científico y tecnológico.

De acuerdo con Maloney, Ramírez, Gunderson, Levine \& Beiloc,(2015), las creencias y actitudes de los padres hacia las matemáticas afectan las creencias y actitudes de los hijos hacia esta materia, si los padres, tienen una percepción negativa, los hijos muy probablemente también lo tendrán. Por ello, es fundamental que los padres sean conscientes del impacto que tienen sus creencias en el desarrollo cognitivo de los hijos. La familia pueden ayudar a desarrollar o matar la curiosidad de los niños hacia el STEM (Early Childhoood STEM Working Group, 2017). Así lo evidencia el estudio "The association of family influence and initial interest in science"en donde preguntaron a estudiantes de doctorado en ciencias físicas ¿qué factores alentaron su interés temprano por la ciencia? El estudio descubrió que la participación familiar facilitaba dicho interés, siendo la ocupación de los padres una fuente primaria de interés (Dabney, Chakraverty \&Tai, 2013).

Diversas investigaciones han demostrado que las relaciones familiares ejercen influencia en la toma de decisiones profesionales en la adolescencia (Garriott, RaqueBogdan, Zoma, Mackie-Hernández, \& Lavin, 2017). Más específicamente, algunos sostienen que la madre y los buenos maestros tienen un impacto mayor en la elección de estudiar carreras STEM en mujeres que en los hombres (Cerinsek, Hribar, Glodez, y Dolinsek, 2013). Esto podría explicarse por la dinámica familiar que se genera en cada hogar, tanto el hombre como la mujer construyen una identidad, adquieren valores y desarrollan intereses con base en los roles asignados dentro del seno familiar. Esto es algo que algunas corrientes de la teoría feminista buscan dilucidar.

En esta perspectiva, a las niñas se les podría enseñar que tienen la capacidad para desarrollar cualquier actividad que se propongan además de formar un hogar y edu- 
car hijos. Las jóvenes tendrían mayor interés en estas disciplinas. Aquí, los profesores adquieren un papel importante como fuentes de motivación para despertar el interés de los estudiantes en carreras STEM sobre todo en las mujeres. Holmes, Gore, Smith y Lloyd (2017), concluyeron en su estudio a 6 mil 492 estudiantes con aspiración de estudiar una carrera STEM que tener un padre en una ocupación STEM resultó significativo para el proceso de elección de los mismos.

A pesar de existir una gran cantidad de investigaciones que muestran que el contexto familiar juega un papel importante en el desarrollo infantil (por ejemplo, Bronfenbrenner, 1986; Jant et al., 2014; Maloney et al., 2015; McCarthy et al., 2015), se han realizado pocas investigaciones sobre como se relacionan los factores familiares en torno al aprendizaje informal, antes que comience la escuela.

En el siguiente apartado se profundiza en la figura de la imagen materna y su influencia en los procesos de elección de los hijos.

\section{El papel de la madre en el desarrollo de la vocación científica}

La madre es pieza fundamental en la formación de los hijos. Sus miedos, frustraciones, deseos, sueños, etc., parte de su ser es transmitido a su descendencia, los cuales adoptan e imitan muchas de las conductas observadas en el hogar.

Tradicionalmente la mujer ha crecido con el rol de ser quien cuida, forma y educa. Sin embargo, estos estereotipos poco a poco han estado cambiando. Cada día crece el número de mujeres profesionistas, e incertas en el mercado laboral. A pesar de ello el porcentaje de mujeres interesadas en estudiar carreras con componente científico o tecnológico sigue siendo menor. La respuesta podría estar en la familia y las madres las posibles responsables de ello. Chandler y Parsons (1995), realizaron el studio The Mother-Daughter Science Club a 20 chicas con sus madres, con el objetivo de despertar el interés de las niñas en ciencia y matemáticas. Al inicio del proyecto, 100 por ciento de las chicas mostraban tener interés en ciencias de la vida, después de nueve semanas de trabajar en los laboratorios y compartir experiencias relacionadas con la ciencia con sus madres, el 25 por ciento de las participantes expresaron su interés en ciencias de la tierra y física. Durante la investigación se dieron cuenta que las madres transmitían a sus hijas sus temores y frustraciones relacionadas con la ciencia y en especial con las matemáticas. Un claro ejemplo de reproducción vocacional.

El estudio de Vázquez y Manassero (2015), en seis países (Argentina, Brasil, Colombia, Panamá, México y España), analizó los factores que influyen en la elección de carreras STEM, uno de los factores fue "personas influyentes" (madre o madrastra, padre o padrastro, buenos profesores, amigos, orientadores vocacionales). Los resultados muestran que para los estudiantes panameños, la persona más influyente es la madre o madrastra, en Brasil, los profesores, seguido de la madre. En Argentina, Colombia, México y España, no hubo diferencias significativas. Sin embargo, en esta investigación se considera a la madre o tutora como un posible factor de influencia, por lo antes descrito.

La familia es un elemento clave en el proceso de elección de carrera de los adolescentes, por lo tanto, una familia que posee un nivel educativo superior podría generar un mayor interés por estas carreras. En la literatura que se revisó poco se ha estudiado esta variable. En su mayoría los estudios se enfocan en el nivel de 
estudio de los padres, pero no en la formación profesional de los mismos. Este factor intergeneracional es necesario estudiarlo más a detalle. Dado que la decisión de elegir una carrera rebasa la escala individual del joven, es necesario, considerar los antecedentes familiares, la formación, profesión e intereses de los padres tendrán un impacto significativo en el proceso de elección de carrera. Aunque parece una decisión autónoma, la mayoría de los estudiantes consideran los antecedentes familiares y la formación para su proceso de elección.

\section{Método}

\section{Objetivo}

El objetivo de la investigación es conocer la influencia de la familia (mamá o tutora, papá o tutor y hermanos) en la elección de carreras STEM en estudiantes de bachillerato. Para dar cumplimiento con el objetivo se analizan las siguientes variables: composición familiar, nivel educativo del padre o tutor, nivel educativo de la madre o tutora, promedio de calificaciones.

\section{Población y Muestra}

La población de análisis fueron estudiantes de segundo y sexto semestre de nivel bachillerato de seis diferentes instituciones educativas de Tabasco, México (COBATAB Plantel 2 y 4, CBTA, CBTIS, CECYTE, CONALEP y PREPA UVM)². La muestra se conformó por 974 estudiantes de segundo y 785 estudiantes de sexto semestre. En total 1 mil 759 participantes, de los cuales, el 55.7 por ciento (979) son hombres y 44.3 por ciento (780) mujeres.

\section{Instrumento}

Se administró el instrumento I-STEM publicado por Avendaño, Magaña y Aguilar (2017), en estudiantes de bachillerato, para conocer la influencia familiar (mamá o tutora, papá o tutor y hermanos) en la elección de carreras STEM. El instrumento está integrado por 26 enunciados, en una escala tipo Likert de 1 a 5 puntos. La dimensión de análisis para esta investigación fue Influencia Familiar.

Se realizó el análisis de fiabilidad (Alpha de Cronbach) para cada dimensión, los cuales de acuerdo con Cortina (1993), los resultados superiores a 7 se consideran buenos y aceptables. Los resultados de esta prueba estadística se presentan en la Tabla 1.

2 Colegio de Bachilleres de Tabasco, Centro de Bachillerato Tecnológico Agropecuario, Centro de Bachillerato Tecnológico Industrial y de Servicios, Colegio de Estudios Científicos y Tecnológicos del Estado de Tabasco, Colegio Nacional de educación Profesional Técnica, Preparatoria Universidad del Valle de México). 
Tabla 1

Análisis de fiabilidad por dimensión

\begin{tabular}{lc}
\hline Dimensión & Alfa \\
\hline Influencia familiar (IF) & .752 \\
Influencia del par académico (IPA) & .805 \\
Aprendizaje no formal (ANF) & .851 \\
Actitudes STEM (ACSTEM) & .899 \\
Profesor/a motivador (PM) & .870 \\
\hline
\end{tabular}

Nota: Elaboración propia

\section{Procedimiento de recogida y análisis de datos}

Para la obtención de los resultados se cuidó cumplir con los criterios éticos establecidos en la investigación. Se solicitó la autorización por escrito de los directores escolares para realizar la administración del cuestionario. Se informó a los estudiantes sobre la importancia de su participación y se garantizó la confidencialidad de los datos.

En la fase de campo, se entregó a cada uno de los participantes un cuestionario impreso, previó al llenado se les proporcionaron las instrucciones correspondientes para cada sección del instrumento, con el objetivo de evitar errores. El tiempo aproximado de respuesta fue de 15 minutos. La administración del cuestionario se realizó en un período de 4 meses de marzo a julio del año 2017.

Para el análisis estadístico, se utilizó el software SPSS versión 21. Se realizó análisis descriptivos, análisis de varianza y pruebas $\mathrm{T}$.

\section{Validez del constructo AFC}

Para llevar a cabo la validez del constructo, se realizó un análisis factorial confirmatorio (AFC) con el método de máxima verosimilitud y Oblimín directo. Se consideraron como índices de ajuste los propuestos por Manzano, Zamora y Salvador (2010) х2, p (ji-cuadrada y probabilidad asociada), TLI (índice de Tucker-Lewis), SRMR (raíz cuadrada de residual estandarizada), CFI (índice de ajuste comparativo), RMSEA IC 90 (error de la raíz cuadrada de la media de aproximación con su intervalo de confianza). Para el caso de los datos de la dimensión (IF), los índices de ajuste fueron aceptables $(\chi 2=7.06, \mathrm{gl}=3, \mathrm{p}=.07 ; \mathrm{TLI}=.97 ; \mathrm{SRMR}=.01 ; \mathrm{CFI}=.99 ; \mathrm{RMSEA}=.02, \mathrm{IC} 90$ [.00 - .05] (Cea, 2005) (ver Figura 1). 


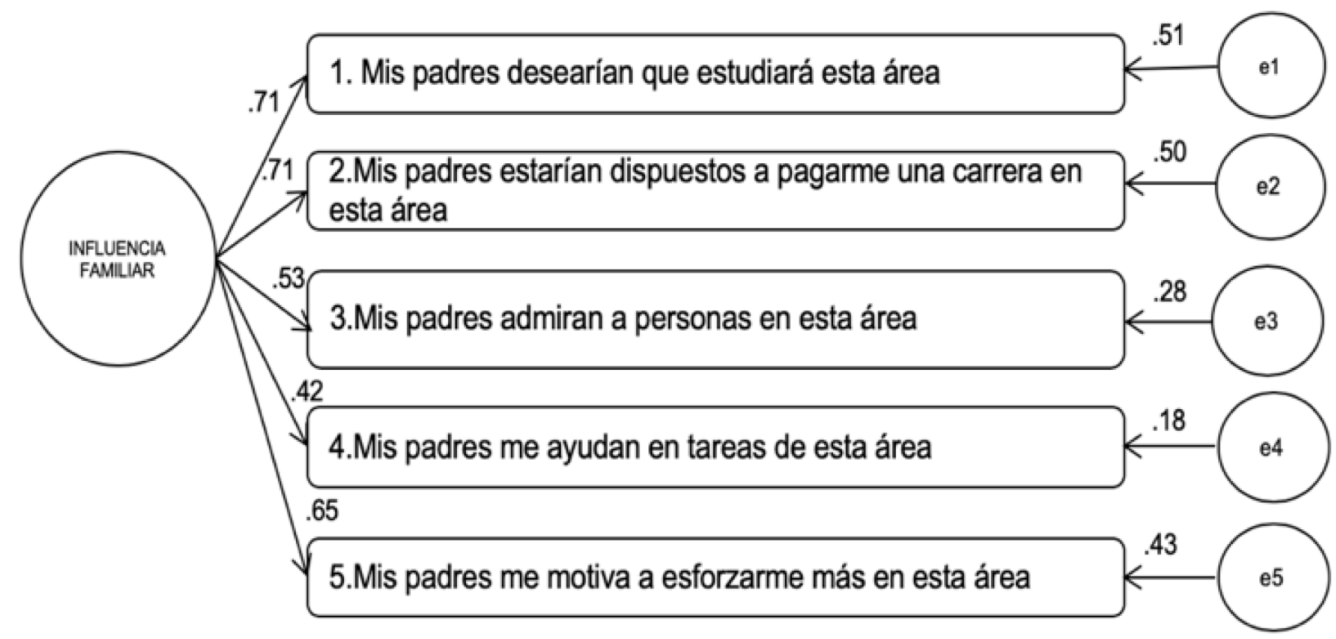

Figura 1. Coeficientes estandarizados y errores estándar del modelo de medición del factor: influencia familiar

\section{Resultados}

En el siguiente apartado se presentan los resultados de los análisis realizados.

\section{Área STEM seleccionada}

El 41.5 por ciento (730) de los estudiantes mostró interés en carreras relacionadas con la ciencia (principalmente ciencias de la salud, química, biología, física) el 30 por ciento (527) en carreras tecnológicas (informática, computación, sistemas), el 23.5 por ciento (420) en el área de ingeniería (civil, mecánica, mecatrónica, geofísica, petrolera, biotecnología) y el 5 por ciento (82) en matemáticas.

En el caso particular de los hombres, el 40 por ciento (392) seleccionó ingeniería como el área de mayor interés, el 28 por ciento (274) optó por disciplinas científicas, el 27 por ciento (264) por tecnología y el 5 por ciento (49) matemáticas. En el caso de las mujeres, el 58 por ciento (453) seleccionó carreras relacionadas con la ciencia, el 20 por ciento (156) ingeniería, el 18 por ciento (140) tecnología y el 4 por ciento (31) matemáticas igual que en hombres, es el área de menor interés.

Era importante conocer aspectos familiares como el nivel educativo de los padres o tutores y la composición familiar. En la Tabla 2. Se presentan las frecuencias y porcentajes de las personas con las que viven los estudiantes. 
Tabla 2

Distribución de frecuencias sobre la composición del núcleo familiar por género

\begin{tabular}{ccccc}
\hline \multirow{2}{*}{ Variables } & \multicolumn{3}{c}{ Género } \\
\cline { 2 - 5 } & \multicolumn{2}{c}{ Hombre } & Fr & Mujer \\
\cline { 2 - 5 } & Fr & \% & 509 & 65.3 \\
\hline Vive con papá o tutor & 697 & 71.2 & 271 & 34.7 \\
No vive con papá o tutor & 282 & 28.8 & 717 & 91.9 \\
Vive con mamá o tutora & 863 & 88.2 & 63 & 8.1 \\
No vive con mamá o tutora & 116 & 11.8 & 96 & 12.3 \\
Vive con abuelos & 166 & 17 & 684 & 87.7 \\
No vive con abuelos & 813 & 83 & 518 & 66.4 \\
Vive con hermanos & 575 & 58.7 & 262 & 33.6 \\
No vive con hermanos & 404 & 41.3 & 60 & 7.7 \\
Vive con otras personas & 70 & 7.2 & & \\
\hline
\end{tabular}

Nota: Elaboración propia

La tabla 2 muestra que más del 60 por ciento de los estudiantes que participaron en la investigación viven con sus padres, lo cual En las conclusiones se profundiza sobre la importancia de vivir con ambos padres y su impacto en la elección de carreras STEM.

Un aspecto de suma importancia para la investigación fue identificar el nivel educativo de los padres o tutores con los que se relaciona de forma cotidiana el estudiante. Los padres con mejor nivel educativo pueden orientar de mejor forma a sus hijos y transmitir un capital cultural mayor (Bourdieu, 1987). Los resultados muestran lo siguiente: el 39 por ciento (599), de los padres o tutores de los estudiantes de bachillerato tiene educación preparatoria, el 25 por ciento (418), estudios universitarios, el 22 por ciento (380), estudios de secundaria, el 10 por ciento (179), estudios de maestría, el 8 por ciento (142), estudios de primaria, el 1 por ciento (20), estudios de doctorado y el 1 por ciento (20) no curso la primaria, pero, sabe leer y escribir.

En la Tabla 3. Se muestra el análisis de varianza para la dimensión de influencia familiar con respecto al tipo de bachillerato. Al analizar las medias, se puede observar que los estudiantes del CONALEP tienen una media mayor con respecto a otras instituciones educativas. Los estudiantes de este subsistema técnico tienen una mayor influencia familiar en la elección de carrera. El tamaño del efecto señala que si puede considerarse un factor que explique las diferencias estadísticas. 
Tabla 3

Análisis de varianza de una vía para la dimensión Influencia Familiar por plantel educativo

\begin{tabular}{|c|c|c|c|c|c|c|}
\hline Variable & Plantel & Media & $D E$ & $F$ & $P$ & $\eta^{2}$ \\
\hline \multicolumn{4}{|c|}{ Influencia Familiar } & 6.55 & .01 & 0.34 \\
\hline & CBTA & 4.30 & 1.06 & & & \\
\hline & CBTIS & 4.44 & 0.95 & & & \\
\hline & CECYTE & 4.34 & 1.09 & & & \\
\hline & PREPA UVM & 4.59 & 1.00 & & & \\
\hline & CETMAR & 4.59 & 0.87 & & & \\
\hline & COBATABP2 & 4.67 & 0.87 & & & \\
\hline & COBATABP4 & 4.33 & 1.06 & & & \\
\hline & CONALEP & 4.74 & 0.87 & & & \\
\hline
\end{tabular}

Nota: $N=1759, p<0.05^{*}, p<0.01^{* *}$

El nivel de estudios de los padres ejerce influencia significativa en la toma de decisiones de sus hijos. Los padres con un nivel de estudios superior y con perfiles STEM tienen conocimientos que permiten orientar mejor al estudiante tal como lo menciona (Dabney et al., 2013).

En la Tabla 4. Se presenta el análisis de varianza sobre la dimensión influencia familiar y el nivel de estudios del padre o tutor. El tamaño del efecto para esta dimensión es relativamente bajo. Habría que estudiar con mayor detalle el rol en el que se desempeña el padre o tutor, así como la formación académica, la investigación únicamente se limitó al nivel de estudio.

Tabla 4

Análisis de Varianza de una vía de la influencia familiar y el nivel de estudios del padre o tutor

\begin{tabular}{llllllc}
\hline Variable & Nivel educativo & Media & $D E$ & $F$ & $P$ & $\eta^{2}$ \\
\hline Influencia familiar & & & 7.13 & 0.01 & 0.03 \\
\hline Ninguna & 3.82 & 1.14 & & & \\
Primaria & 4.23 & 0.95 & & & \\
Secundaria & 4.38 & 1.06 & & & \\
Bachillerato & 4.47 & 0.97 & & & \\
Licenciatura & 4.63 & 0.96 & & & \\
Maestría & 4.73 & 0.92 & & & \\
Doctorado & 4.40 & 1.06 & & & \\
\hline
\end{tabular}

Nota: $N=1759, p<0.05^{*}, p<0.01^{* *}$ 
De igual forma se realizó el análisis de varianza para la madre o tutora. La Tabla 5. Muestra el tamaño del efecto para esta dimensión, el cual también es bajo.

Tabla 5

Análisis de Varianza de una vía de la influencia familiar y el nivel de estudios de la madre o tutora

\begin{tabular}{lllllll}
\hline Variable & Nivel educativo & Media & $\mathbf{D E}$ & $\boldsymbol{F}$ & $\boldsymbol{P}$ & $\boldsymbol{\eta}^{2}$ \\
\hline Influencia familiar & & & 6.61 & 0.01 & .03 \\
\hline Ninguna & 4.27 & 1.12 & & & \\
Primaria & 4.23 & 1.03 & & \\
Secundaria & 4.36 & 1.03 & & \\
Bachillerato & 4.53 & 0.97 & & \\
Licenciatura & 4.69 & 0.98 & & \\
Maestría & 4.63 & 0.80 & & \\
Doctorado & 4.30 & 0.93 & & & \\
\hline
\end{tabular}

Nota: $N=1759, p<0.05^{*}, p<0.01^{* *}$

En otro tenor, la Tabla 6. Presenta el análisis de varianza de la dimensión influencia familiar y el rango promedio de calificaciones, el tamaño del efecto es mínimo.

Tabla 6

Tabla de Análisis de Varianza de una vía de la influencia familiar y el rango promedio de calificación

\begin{tabular}{lllllll}
\hline Variable & $\begin{array}{c}\text { Promedio de } \\
\text { calificaciones }\end{array}$ & Media & DE & $\boldsymbol{F}$ & $\boldsymbol{P}$ & $\boldsymbol{\eta}^{2}$ \\
\hline Influencia familiar & & & 4.73 & 0.01 & .02 \\
\hline $6.0-6.9$ & 4.27 & 1.12 & & & \\
$7.0-7.5$ & 4.23 & 1.03 & & \\
$7.6-8.1$ & 4.36 & 1.03 & & \\
$8.2-8.7$ & 4.53 & 0.97 & & \\
$8.8-9.3$ & 4.69 & 0.98 & & \\
$9.4-10$ & 4.63 & 0.80 & & \\
\hline
\end{tabular}

Nota: $N=1759, p<0.05^{*}, p<0.01^{* *}$

Se realizó prueba T de Student para la dimensión influencia familiar y si vive o no con su padre o tutor. El tamaño del efecto es pequeño, pero significativo. 
Tabla 7

Diferencias entre los grupos que viven con su padre o tutor y los que no, con relación a la influencia familiar

\begin{tabular}{|c|c|c|c|c|c|c|c|}
\hline \multirow[t]{2}{*}{ Variable } & \multicolumn{2}{|c|}{$\begin{array}{c}\text { Vive con su padre } \\
\text { o tutor } \\
\end{array}$} & \multicolumn{2}{|c|}{$\begin{array}{l}\text { No vive con su } \\
\text { padre o tutor }\end{array}$} & \multirow[t]{2}{*}{$T$} & \multirow[t]{2}{*}{$P$} & \multirow{2}{*}{$\begin{array}{l}d \text { de } \\
\text { Cohen }\end{array}$} \\
\hline & $M$ & $S D$ & $M$ & $S D$ & & & \\
\hline Influencia familiar & 4.53 & 0.97 & 4.40 & 1.04 & 2.46 & $0.01^{* *}$ & 0.12 \\
\hline
\end{tabular}

Nota: $N=1757, p<0.05^{*}, p<0.01^{* *}$

Al igual que con el padre o tutor se realizó el análisis de la dimensión influencia familiar con relación a si vive o no con su madre o tutora. A diferencia del padre, el tamaño del efecto en la madre es mayor que el del padre. La presencia de la madre en el seno familiar es sumamente importante ya que influye en muchas de las decisiones. Al parecer no influye tanto el nivel educativo de la madre, pero si su presencia en la familia, este es uno de los hallazgos encontrados en la investigación.

Tabla 8

Diferencias entre los grupos que viven con su madre o tutora y los que no, con relación a la influencia familiar

\begin{tabular}{|c|c|c|c|c|c|c|c|}
\hline \multirow{2}{*}{ Dimensiones } & \multicolumn{4}{|c|}{$\begin{array}{cc}\begin{array}{c}\text { Vive con su madre } \\
\text { o tutora }\end{array} & \begin{array}{c}\text { No con su madre } \\
\text { o tutora }\end{array} \\
\end{array}$} & \multirow{2}{*}{$T$} & \multirow[t]{2}{*}{$P$} & \multirow{2}{*}{$\begin{array}{l}d \text { de } \\
\text { Cohen }\end{array}$} \\
\hline & $\mathbf{M}$ & SD & $\mathbf{M}$ & SD & & & \\
\hline Influencia familiar & 4.51 & 0.99 & 4.30 & 1.03 & 2.65 & $0.00^{* *}$ & .20 \\
\hline
\end{tabular}

Nota: $N=1757, p<0.05^{*}, p<0.01^{* *}$

En el siguiente apartado se presenta una serie de reflexiones y conclusiones a partir de los resultados de la investigación.

\section{Discusión y conclusiones}

El proceso de elección de carrera es si duda una de las decisiones más importantes en la vida de los sujetos. Es el momento cuando el individuo motivado por factores internos y externos elige el rumbo de su vida. Los llamados factores externos, como la familia, amigos, profesores, medios de comunicación, redes sociales, etc., influyen de manera directa en los intereses y motivaciones internas del sujeto, quien, influenciado por su microcosmo, desarrolla un fuerte interés en aquello que logra despertar sus deseos internos, los más profundos de su ser (Watt, \& Richardson, 2007).

La familia juega un papel importe en los procesos de crecimiento de los sujetos, es en el seno familiar, donde se le provee de conocimientos primarios, valores, creencias, 
se trasmiten costumbres y tradiciones que formarán su propia identidad y cultura, a partir del capital cultural familiar, tal como lo plantea Bourdieu en (1987).

De acuerdo con la teoría social cognitiva de carrera propuesta por Lent, Brown \& Hackett (1994), el individuo recibe constantemente múltiples estímulos exteriores que moldean su carácter e intereses, al contacto con aquello que lo estimula, despierta su interés, curiosidad, deseo de saber, lo toma, adquiere y forma parte de si. Las vocaciones, son motivadas e influenciadas por los propios intereses de los sujetos con los que convive el individuo, cuando este adquiere cierto nivel de conciencia se siente con el poder de elegir aquello que lo hace feliz, en lo que se siente realizado, útil, encuentra su verdadera vocación, para lo que fue creado (Rehm, 1990). Sin embargo, en la medida que crece, aprende, cuestiona, se trasforma o ratifica sus propios gustos e intereses, con mayor nivel de conciencia y racionalidad el sujeto debe enfrentarse a una de las elecciones más trascendentales de su vida ¿qué carrera elegir? ¿a quién debo escuchar? ¿cuál es el papel de la familia en este proceso y como influye en la elección? En la investigación se analizó la influencia de la familia como un factor que interviene en la toma de decisiones de los sujetos. Es en la familia donde los hijos construyen una primera visión de la realidad, se reconocen y asocian a un determinado grupo de personas, llamado familia, que constituye la base más importante del sujeto (Bratcher, 1982). Habiendo dicho lo anterior, los antecedentes familiares, la formación profesional, intereses familiares, pasiones, hobbies, y su vinculación con la ciencia y tecnología son factores que ejercen influencia en los sujetos (Sheehan, Hightowe, Lauricella \& Wartella, 2018). En la investigación no sé profundizó en estos aspectos familiares. Es necesario que el tema sea abordado en próximos estudios de forma más profunda.

Los resultados de la investigación muestran que vivir con el padre o tutor tiene un efecto mínimo en el proceso de elección de carrera, caso contrario a la madre o tutora, el efecto es significativamente mayor. Los estudiantes que viven con su madre o tutora ven en ella una figura de autoridad, además el vínculo es mucho más profundo con ella, por lo tanto, lo que opine, exprese o piense cobra importancia para el sujeto (Watt, Hyde, Petersen, Morris \& Harackiewicz, 2017). Vivir con la madre o tutora es un elemento significativo en los procesos de decisión del sujeto ¿entonces, ¿qué tanto influye el nivel de estudios de los padres en la elección de carrera? Los resultados de la investigación mostraron un efecto mínimo, se contraponen a la investigación de Riegle-Crumb y King (2010), quienes concluyen que el nivel educativo de los padres, cultura, gustos, motivación e intereses son factores que tiene un impacto en la elección de carrera, habrá que profundizar en este aspecto en las siguientes investigaciones. Por otro lado, el trabajo de Harackiewicz, Rozek, Hulleman, y Hyde (2012), aunque no sé enfocó en investigar el nivel educativo, descubrió que el conocimiento que poseen los padres sobre STEM es limitado. Esto mismo podría estar ocurriendo en el contexto nacional.

Por otro lado, resulta interesante analizar lo que ocurre con los estudiantes de familias con niveles socioeconómicos bajos y en desventaja social. La evidencia muestra que tienen mayor interés en carreras científicas y tecnológicas ya que encuentran en estas carreras la posibilidad de lograr mayor movilidad social (Chen, 2009). No obstante, en el futuro se pueden conducir estudios en dos sentidos. En primer lugar, si estos jóvenes tienen la @libertad® de alcanzar estos objetivos individualmente razonados. 
En segundo lugar, habrá que conducir investigaciones en zonas rurales y urbanas que permitan identificar y comparar los niveles de interés en las disciplinas STEM entre los estudiantes y sus antecedentes familiares.

La sociedad se encuentra en un momento de transformación intelectual, cultural, tecnológica, científica y social que ha impactado fuertemente la composición y funcionamiento familiar. Es necesario que se profundice en los estudios de género y en la concepción moderna de la mujer, sus nuevos roles dentro de la familia, sus intereses, motivaciones y aspiraciones de vida (Barnett \& Hyde, 2001). Hoy, la mujer ocupa posiciones de poder, es protagonista en los negocios, arte, política, deportes, cultura y ciencia. Aunque el porcentaje de mujeres científicas sigue siendo bajo comparado con el de los hombres (Organización de las Naciones Unidas para la Educación, la Ciencia y la Cultura (UNESCO), 2019) . El involucramiento de la mujer es cada vez mayor. Los roles socialmente establecidos están siendo desafiados por nuevas generaciones que buscan mayor igualdad y realización profesional, pero¿qué tan interesadas están las mujeres en las disciplinas STEM? ellas se interesan en carreras relacionadas con la ciencia. Beede, Julian, Langdon, McKittrick, Khan y Doms (2011), en su investigación hacen notar que las mujeres están mas interesadas en ciencias de la salud (e.g. medicina y enfermería). Los resultados coinciden con los obtenidos en la investigación, el 57 por ciento de las estudiantes encuestadas mencionaron ciencia como su primera opción de estudio. Las mujeres evitan elegir carreras que limitan su desarrollo como madres o que representan largas horas de trabajo (Guillaume \& Pochic, 2009). Quizá si el sistema permitiera a las mujeres su desarrollo como madres y horarios laborales más flexibles se pudiera beneficiar de su talento y conocimientos (Gregory \& Milner, 2009; Emslie \& Hunt, 2009). Aunque la presencia femenina en el mercado laboral ๑masculino॰ va en aumento, sigue siendo minoría (Kier, Blanchard, Osborne, y Albert, 2014). Es necesario cambiar la visión y contribuir en la contrucción de un sistema educativo que garantice las mismas oportunidades entre hombres y mujeres y propicie el involucramiento de las mujeres en áreas consideradas socialmente como masculinas. La familia moderna esta frente a la gran responsabilidad de formar mujeres y hombres empoderados, con valores, conocimientos y nuevas concepciones de vida.

En general el tema de la elección de carreras STEM y los factores asociados al proceso deben ser estudiados a profundidad. Comprender en su totalidad desde diversos enfoques metodológicos lo que ocurre con la familia, el impacto que tienen las relaciones psicosociales en la elección de los jóvenes, son temas pendientes. Por otro lado, se recomienda realizar investigaciones más profundas con las madres e hijas con el objetivo de identificar los factores y comportamientos que motivan o restringen el acceso de las mujeres en áreas STEM.

La investigación deja abierta la pregunta ¿cómo elegirán su carrera profesional los nacidos en la generación Z? ¿qué tan importante será la familia en la elección? Bien o mal los millenials han realizado sus procesos de elección, influenciados por factores tradicionales, como la familia, algunos por vocación, profesores, contexto, etc., Pero, esta nueva generación llena de jóvenes irreverentes, nativos digitales, fuertemente influenciados por las redes sociales, influencers juveniles ¿cómo realizarán sus procesos de elección profesional? Es necesario plantearnos nuevas preguntas en torno a una nueva generación, que nos obliga a comprender sus nuevas formas de pensamiento, 
motivaciones, intereses y pasiones profesionales. Los estudios longitudinales pueden ayudar a responder estás interrogantes. Esta decisión impacta en la estructura económica, en la innovación y desarrollo de un país. Es urgente profundizar en el debate.

\section{Referencias}

Avendaño, Rodríguez, K.C, Magaña, Medina, D. E., \& Aguilar, Morales, N. (2017). Análisis factorial exploratorio del cuestionario interés por estudios universitarios en áreas STEM (I-STEM). Revista de Análisis Cuantitativo y Estadístico, 4(13), 54-68.

Bagiati, A., Yoon, S. Y., Evangelou, D., \& Ngambeki, I. (2010). Engineering Curricula in Early Education: Describing the Landscape of Open Resources. Early Childhood Research \& Practice, 12(2), n2.

Barnett, R. C., \& Hyde, J. S. (2001). Women, men, work, and family: An expansionist theory. American psychologist, 56(10), 781.

Beede, D. N., Julian, T. A., Langdon, D., McKittrick, G., Khan, B., \& Doms, M. E. (2011). Women in STEM: A gender gap to innovation.

Bourdieu, P. (1987). What makes a social class? On the theoretical and practical existence of groups. Berkeley journal of sociology, 32, 1-17.

Bourdieu, P. (1997). Capital cultural, escuela y espacio social. Coyoacán, México, Siglo XXI.

Bratcher, W. E. (1982). The influence of the family on career selection: A family systems perspective. The Personnel and Guidance Journal, 61(2).

Breiner, J. M., Harkness, S. S., Johnson, C. C., \& Koehler, C. M. (2012). What is STEM? A discussion about conceptions of STEM in education and partnerships. School Science and Mathematics, 112(1), 3-11.

Bronfenbrenner, U. (1986). Ecology of the family as a context for human development: Research perspectives. Developmental psychology, 22(6), 723.

Cerinsek, G., Hribar, T., Glodez, N., \& Dolinsek, S. (2013). Which are my future career priorities and what influenced my choice of studying science, technology, engineering or mathematics? Some insights on educational choice-case of Slovenia. International Journal of Science Education, 35(17), 2999-3025.

Chandler, F. T., \& Parsons, S. (1995). A Case Study of a Mother/Daughter Science Club.

Chen, X. (2009). Students Who Study Science, Technology, Engineering, and Mathematics (STEM) in Postsecondary Education. Stats in Brief. NCES 2009-161. National Center for Education Statistics.

Cortina, J. M. (1993). What is coefficient alpha? An examination of theory and applications. Journal of applied psychology, 78(1), 98.

Dabney, K. P., Chakraverty, D. and Tai, R. H. (2013). The association of family influence and initial interest in science. Science Education, 97(3), 395-409.

Early Childhood STEM Working Group. (2017). Early STEM matters: Providing highquality STEM experiences for all young learners. Chicago (IL): UChicago STEM Education and Erikson Institute.

Emslie, C., \& Hunt, K. (2009). 'Live to work'or 'work to live'? A qualitative study of gender and work-life balance among men and women in mid-life. Gender, Work $\mathcal{E}$ Organization, 16(1), 151-172. 
Garriott, P. O., Raque-Bogdan, T. L., Zoma, L., Mackie-Hernandez, D., \& Lavin, K. (2017). Social cognitive predictors of Mexican American high school students' math/ science career goals. Journal of Career Development, 44(1), 77-90.

Gregory, A., \& Milner, S. (2009). Work-life balance: A matter of choice?. Gender, Work \& Organization, 16(1), 1-13.

Guillaume, C., \& Pochic, S. (2009). What would you sacrifice? Access to top management and the work-life balance. Gender, Work and Organization, 16(1), 14-36.

Han, X., \& Appelbaum, R. P. (2018). China's science, technology, engineering, and mathematics(STEM) research environment: A snapshot. PloS one, 13(4).

Harackiewicz, J. M., Rozek, C. S., Hulleman, C. S., \& Hyde, J. S. (2012). Helping parents to motivate adolescents in mathematics and science: An experimental test of a utility-value intervention. Psychological Science, 23(8), 899-906.

Holmes, K., Gore, J., Smith, M., \& Lloyd, A. (2017). An integrated analysis of school students' aspirations for STEM careers: Which student and school factors are most predictive? International Journal of Science and Mathematics Education, 16(4), 655-675.

Jant, E. A., Haden, C. A., Uttal, D. H. and Babcock, E. (2014). Conversation and object manipulation influence children's learning in a museum. Child development, 85(5), 2029-2045.

Kier, M. W., Blanchard, M. R., Osborne, J. W., \& Albert, J. L. (2014). The development of the STEM career interest survey (STEM-CIS). Research in Science Education, 44(3), 461-481.

Langdon, D., McKittrick, G., Beede, D., Khan, B., \& Doms, M. (2011). STEM: Good Jobs Now and for the Future. ESA Issue Brief\# 03-11. US Department of Commerce.

Lent, R. W., Brown, S. D., \& Hackett, G. (1994). Toward a unifying social cognitive theory of career and academic interest, choice, and performance. Journal of vocational behavior, 45(1), 79-122.

Maloney, E. A., Ramirez, G., Gunderson, E. A., Levine, S. C. and Beilock, S. L. (2015). Intergenerational effects of parents' math anxiety on children's math achievement and anxiety. Psychological Science, 26(9), 1400-1488.

Manzano, A., Zamora, S., \& Salvador, R. (2010). Sistema de ecuaciones estructurales: una herramienta de investigación. Ciudad de México.

McCarthy, B., Li, L., Tiu, M., Atienza, S. and Sexton (2015). Learning with PBS KIDS: A study of family engagement and early mathematics achievement.

Nadelson, L. S., \& Seifert, A. L. (2017). Integrated STEM defined: Contexts, challenges, and the future. Journal of Educational Research, 110(3), 221-223.

National Academy of Sciences. (2014). STEM Integration in K-12 Education: Status, Prospects, and an Agenda for Research. Washington, D.C.: The National Academy of Sciences. doi.10.17226/18612

National Science Board. (2016). Science and Engineering Indicators 2016. National Science Board, 897.

Organización de las Naciones Unidas para la Educación, la Ciencia y la Cultura (UNESCO). (2019). Women in Science. http://uis.unesco.org/sites/default/files/ documents/fs55-women-in-science-2019-en.pdf

Organización para la Cooperaión y el Desarrollo Económico (OCDE) /Comisión Económica para América Latina y el Caribe (CEPAL) /Banco de Desarrollo de América 
Latina (CAF). (2015). Perspectivas Económicas De América Latina 2016. Hacia una nueva asociación con China. París, Francia: OECD Publishing.

Peterson, A., Gaskill, M., \& Cordova, J. (2018). Connecting STEM with Social Emotional Learning (SEL) Curriculum in Elementary Education. In Society for Information Technology E Teacher Education International Conference .1212-1219. Association for the Advancement of Computing in Education (AACE). Recuperado de https://www. learntechlib.org/primary/p/182681/

Rehm, M. (1990). Vocation as personal calling: A question for education. The Journal of Educational Thought (JET)/Revue de la Pensée Educative, 114-125.

Riegle-Crumb, C., \& King, B. (2010). Questioning a white male advantage in STEM: Examining disparities in college major by gender and race/ethnicity. Educational Researcher, 39(9), 656-664.

Rojko, A. (2017). Industry 4.0 concept: background and overview. International Journal of Interactive Mobile Technologies (iJIM), 11(5), 77-90.

Sheehan, K. J., Hightower, B., Lauricella, A. R., \& Wartella, E. (2018). STEM Media in the Family Context: The Effect of STEM Career and Media Use on Preschoolers' Science and Math Skills. European Journal of STEM Education, 3(3), 17.

Toma, R. B., \& Greca, I. M. (2018). The effect of integrative STEM instruction on elementary students' attitudes toward science. Eurasia Journal of Mathematics, Science and Technology Education, 14(4), 1383-1395.

Vázquez Alonso, Á., \& Manassero Más, M. A. (2015). La elección de estudios superiores científico-técnicos: análisis de algunos factores determinantes en seis países. Revista Eureka sobre Enseñanza y Divulgación de las Ciencias, 12(2), 264. doi: 10498/17251

Watt, H. M., \& Richardson, P. W. (2007). Motivational factors influencing teaching as a career choice: Development and validation of the FIT-Choice scale. The Journal of experimental education, 75(3), 167-202.

Watt, H. M., Hyde, J. S., Petersen, J., Morris, Z. A., Rozek, C. S., \& Harackiewicz, J. M. (2017). Mathematics - A critical filter for STEM-related career choices? A longitudinal examination among Australian and US adolescents. Sex Roles, 77(3-4), 254-271.

Fecha de recepción: 6 de marzo de 2019.

Fecha de revisión: 23 de abril de 2019.

Fecha de aceptación: 10 de marzo de 2020. 
